\title{
Grenzüberschreitende multimodale Güterbeförderung in der EU: Internationale Gerichtszuständigkeit und anwendbares Vertragsrecht - Ein Überblick
}

\author{
Simon Laimer
}

\section{Einleitung: Multimodaler Güterbeförderungsvertrag mit Auslandsberührung}

Eine nachhaltigere Gestaltung des Verkehrssektors stellt, nicht zuletzt auch mit Blick auf die Umsetzung des Pariser Klimaschutzabkommens, ein zentrales Anliegen der EU-Verkehrspolitik dar. ${ }^{1}$ Dabei ist ein wesentliches Element der EUStrategie für eine umweltfreundliche Mobilität ${ }^{2}$ - etwa neben der Steigerung der Effizienz des Verkehrssystems durch Digitalisierung ${ }^{3}$ - vor allem eine bedeutende Verlagerung des Güterverkehrs von der Straße auf emissionsärmere Verkehrsträger wie insbesondere den Schienenverkehr. Damit sind Umweltschutzziele, aber auch die erstrebte Entlastung der Straßenverkehrswege gemeinsam mit der immer weiter reichenden Einführung von normierten Transportgefäßen (speziell der Containerverkehr) ganz wesentliche Gründe dafür, dass Gütertransportvorgänge zunehmend durch eine Aufeinanderfolge verschiedener Transportmittel gekennzeichnet sind. ${ }^{4}$

\footnotetext{
${ }^{1}$ So ausdrücklich die Begründung der Europäischen Kommission zu ihrem Vorschlag für eine Richtlinie „zur Änderung der Richtlinie 92/106/EWG über die Festlegung gemeinsamer Regeln für bestimmte Beförderungen im kombinierten Güterverkehr zwischen Mitgliedstaaten“, $\operatorname{KOM}(2017) 648$ endg/2 (S. 2).

${ }^{2}$ Siehe dazu die Mitteilung der Europäischen Kommission über „Eine europäische Strategie für emissionsarme Mobilität", KOM(2016) 501 endg.

${ }^{3}$ Vgl. hierzu etwa Furrer/Brugger, UNCITRAL Model Law on Electronic Transferable Records Ansatz für die Rechtvereinheitlichung der Schnittstelle zwischen papiergebundenen und elektronischen Dokumenten, in Festschrift für Thomas Koller (2018) 215 ff.

${ }^{4}$ Jesser-Huß, Multimodaler Transport, in Basedow/Hopt/Zimmermann (Hrsg.), Handwörterbuch des Europäischen Privatrechts II (2009) 1087; Mankowski, in Reithmann/Martiny (Hrsg.), Interna-
}

\footnotetext{
S. Laimer $(\bowtie)$

Institut für Zivilrecht, Johannes Kepler Universität Linz, Linz, Österreich

E-Mail: simon.laimer@jku.at
}

S. Laimer, C. Perathoner (Hrsg.), Mobilitäts- und Transportrecht in Europa,

Bibliothek des Wirtschaftsrechts 2,

https://doi.org/10.1007/978-3-662-63635-0_2 
So wird beispielsweise eine Sendung, die von Stockholm nach Bozen befördert wird, zunächst in einen Lastwagen verladen, der sie zu einem Flughafen für die Verladung auf ein Flugzeug (oder etwa zu einem Hafen für die Verladung auf ein Schiff) bringt. Das Flugzeug wird die Ladung etwa nach Mailand (oder das Schiff z. B. nach Genua) bringen, wo sie wiederum auf einen LKW oder eben auf einen Bahnwagon verladen wird, mit dem sie schließlich nach Bozen gebracht wird. ${ }^{5}$

Werden in solchen Fällen selbstständige Beförderungsverträge über die einzelnen Teilstrecken geschlossen (sog. gebrochener bzw. segmentierter Verkehr), dann kommen grundsätzlich die jeweiligen Regelungen des zumeist nach den verschiedenen Verkehrsträgern unterscheidenden Transportrechts zur Anwendung. ${ }^{6}$ Dagegen handelt es sich um einen sog. multimodalen Transport, wenn er mit mindestens zwei verschiedenen Transportmitteln ${ }^{7}$ unter der Verantwortung eines Gesamtbeförderers (Multimodal Transport Operator - MTO) auf Grund eines einheitlichen Beförderungsvertrages durchgeführt wird. ${ }^{8}$ Dieser „Durchfrachtvertrag“ regelt den Gütertransport also vom Übernahme- bis zum Bestimmungsort und bis zur Auslieferung an den Empfänger. ${ }^{9}$ Zuweilen war in diesem Zusammenhang auch der Begriff des „kombinierten“ Transports (oder Verkehrs) verbreitet. ${ }^{10}$

Wird die Förderung multimodaler Lösungen im Güterverkehr als Ziel der EU-Verkehrspolitik formuliert, so ist (neben Infrastrukturmaßnahmen oder technischer Normung) die erforderliche Harmonisierung des ordnungspolitischen Rahmens und hier insbesondere auch der Bereich der privatrechtlichen Rahmenbedingungen angesprochen. Nachdem der betroffene Rechtsbereich wirtschaftlich stark grenzüberschreitend ausgerichtet ist, sollen im folgenden Überblick jene besonderen Fragen für Gütertransporte innerhalb der EU in den Mittelpunkt gestellt werden, die speziell mit Blick auf Haftungsstreitigkeiten aus dem multimodalen Beförderungsvertrag (etwa wegen Beschädigung oder Verlust des Transportgutes) dann auftreten, wenn der Übernahmeort des Gutes und der Ort der Ablieferung in zwei verschiedenen Staaten liegen oder ggf. auch dann gelöst werden müssen, wenn die Vertragsparteien ihre Niederlassungen in unterschiedlichen Staaten haben. ${ }^{11}$ In der-

\footnotetext{
tionales Vertragsrecht ${ }^{8}$ (2015) Rz 6.2091.

${ }^{5}$ Siehe auch Maurer, Einheitsrecht im internationalen Warentransport, RabelsZ 81 (2017) 117 (146).

${ }^{6}$ Von Hoffmann, in Soergel, Bürgerliches Gesetzbuch mit Einführungsgesetz und Nebengesetzen ${ }^{12}$ (1996) Art. 28 EGBGB Rz 469; Buonocore, I contratti di trasporto e di viaggio (2003) 204.

${ }^{7}$ Bei ,aufeinander folgenden Frachtführern“ (z. B. nach Art. 34 CMR) ändert sich die Transportart hingegen nicht, weshalb kein multimodaler Vertrag vorliegt, Mankowski, in Reithmann/Martiny Rz 6.2097.

${ }^{8}$ Spickhoff, in BeckOK BGB ${ }^{49}$. Ed. (2019) VO (EG) 593/2008 Art. 5 Rz 12; Staudinger, in Schulze u. a. (Hrsg.), Bürgerliches Gesetzbuch - Handkommentar ${ }^{10}$ (2019) Art. 5 Rom I-VO Rz 5 m. w. N. ${ }^{9}$ Martiny, in Münchener Kommentar zum Bürgerlichen Gesetzbuch ${ }^{7}$ (2018) Rom I-VO Art. $5 \mathrm{Rz} 140$.

${ }^{10}$ Jesser-Huß, in Handwörterbuch II, 1087, auch zum Begriff des intermodalen Transports, der nur solche Beförderungen bezeichnet, bei denen Güter in intermodalen Ladeeinheiten oder einem Straßenfahrzeug befördert und mit diesem umgeladen werden.
}

${ }^{11}$ Mankowski, in Reithmann/Martiny Rz 6.2093. 
artigen grenzüberschreitenden Fällen ist nämlich zuvorderst zu klären, welche Gerichte für den Streit international zuständig sind und welches materielle Recht für die Entscheidung maßgeblich ist.

\section{Der Rechtsrahmen und seine Grenzen}

Grundsätzlich haben die Bemühungen zur Rechtsvereinheitlichung im Bereich der internationalen Gütertransporte auf der Straße, ${ }^{12}$ mit der Eisenbahn, ${ }^{13}$ auf Binnengewässern, ${ }^{14}$ im Luftverkehr ${ }^{15}$ sowie - eingeschränkter - auf See ${ }^{16}$ mittlerweile weitreichende Früchte getragen. ${ }^{17}$ Mit den Übereinkommen wurde somit eine Vereinheitlichung in praktisch wichtigen Rechtsfragen des grenzüberschreitenden

\footnotetext{
${ }^{12}$ Übereinkommen über den Beförderungsvertrag im internationalen Straßengüterverkehr (CMR) v. 19.5.1956, samt Protokoll v. 5.7.1978. Vgl. Carr/Kidner, International Trade Law Statutes and Conventions 2009-2010 (2010) 757 ff.; Laimer, International Carriage of Goods by Road between Uniform Law and Rome I: Some Recent Austrian Cases, EuLF 3/4 (2017) 58 ff. Zur Situation in Amerika vgl. Larsen, The Uniform Legal Regime Governing Inter-American Contracts for Carriage of Goods by Road, Unif. L. Rev. 2002, Vol. 7(3), 791 ff.

${ }^{13}$ Übereinkommen über den internationalen Eisenbahnverkehr (COTIF) v. 9.5.1980 i. d. F. des Änderungsprotokolls v.3.6.1999, mit dem Anhang B: Einheitliche Rechtsvorschriften für den Vertrag über die internationale Eisenbahnbeförderung von Gütern (CIM) v. 9.6.1999.

${ }^{14}$ Budapester Übereinkommen über den Vertrag über die Güterbeförderung in der Binnenschifffahrt (CMNI) v. 22.6.2001.

${ }^{15}$ Übereinkommen zur Vereinheitlichung bestimmter Vorschriften über die Beförderung im internationalen Luftverkehr (Montrealer Übereinkommen - MÜ) v. 28.5.1999. Zum Verhältnis zwischen MÜ und EU-Sekundärrecht siehe EuGH 10.01.2006, Rs. C-344/04, IATA und ELFAA, ECLI:EU:C:2006:10 (Rz 35 ff.). Staaten, die das MÜ nicht ratifiziert haben, wenden weiterhin das Abkommen zur Vereinheitlichung von Regeln über die Beförderung im Internationalen Luftverkehr (Warschauer Abkommen) v. 12.10.1929 an.

${ }^{16}$ Insb. Internationales Abkommen zur Vereinheitlichung von Regeln über Konnossemente v. 25.8.1924 (Haager Regeln); Änderungsprotokoll v. 23.2.1968 (Visby Regeln); Internationales Übereinkommen zur einheitlichen Feststellung einzelner Regeln über die Konnossemente v. 25.8.1924 i. d. F. des Protokolls v. 23.2.1968 sowie des Protokolls v. 21.12.1979 (Haager-Visby Regeln); Übereinkommen der Vereinten Nationen über die Beförderung von Gütern auf See v. 31.3.1978 (Hamburg Regeln); Übereinkommen der Vereinten Nationen über Verträge über die internationale Beförderung von Gütern ganz oder teilweise auf See v. 23.11.2009 (Rotterdam Regeln). Vgl. Furrer, Das neue Seehandelsrecht im Kontext internationaler und privater Rechtsvereinheitlichung, RdTW 2014, 85 ff.; Djadjev, Law and Practice of the Obligations of the Carrier over the Cargo - The Hague-Visby Rules (2016); Salmerón Henríquez, Freedom of Contract, Bargaining Power and Forum Selection in Bills of Lading (2016) 327 ff.; v.Ziegler/Schellenberg, Die Rotterdam Regeln - Werdegang und Einführung, in Ramming (Hrsg.), Die Rotterdam Regeln (2011) 1 ff. Zum Personentransport Kindler, Die Haftung von Beförderern für Schäden von Reisenden bei Unfällen auf See: die Haftungshöchstgrenzen des HGB und der Verordnung (EG) Nr. 392/2009 im Lichte des höherrangigen Rechts, TranspR 2019, 153 ff.
}

${ }^{17}$ Maurer, RabelsZ 81 (2017) 127 ff. 
Güterfernverkehrs, unter anderem etwa zu Gerichtszuständigkeiten und zur Vertragshaftung, erzielt. ${ }^{18}$

Allerdings sind die unterschiedlichen internationalen Rechtsvorschriften jeweils nur für Transporte mit ein und demselben Beförderungsmittel (also unimodal) konzipiert, sodass sie grundsätzlich nicht direkt zur Anwendung gelangen, wenn ein Gut während einer einheitlichen Beförderung mit mehreren verschiedenen Verkehrsträgern (mithin multimodal) transportiert wird. ${ }^{19}$ Die Versuche, für die internationale multimodale Güterbeförderung vereinheitlichte Regelungen zu schaffen, sind bislang auch deshalb fehlgeschlagen, weil die Vertreter der Teiltransportrechte nicht bereit sind, von ihren erreichten Positionen abzurücken. ${ }^{20}$ So darf auch die 1980 verabschiedete einschlägige Konvention der Vereinten Nationen ${ }^{21}$ mangels ausreichender Ratifikationen mittlerweile als gescheitert betrachtet werden. ${ }^{22}$

Ausnahmen bilden lediglich einzelne Sondervorschriften in den unimodalen Transportrechtsübereinkommen, wie etwa Art. 2 CMR für den sog. Huckepacktransport, bei welchem das mit dem Gut beladene Fahrzeug auf einer Teilstrecke durch ein anderes Verkehrsmittel befördert wird, ohne dass eine Umladung erfolgt (z. B. ,Rollende Landstraße“, ${ }^{23}$ Ro/Ro-Verkehr, Fährschifftransport). ${ }^{24} \mathrm{Zu}$ nennen ist auch Art. 1 \& 3 CIM, der den Straßenvor- oder -nachlauf zur Bahnbeförderung

\footnotetext{
${ }^{18}$ Mankowski, in Reithmann/Martiny Rz 6.1922.

${ }^{19}$ Csoklich, OGH zum multimodalen Transport und zu zwei weiteren transportrechtlichen Fragen, RdW 1994, 339; Mankowski, Anmerkung zu EuGH 11.07.2018 - C-88/17, TranspR 2018, 474 ff.; Rogert, Einheitsrecht und Kollisionsrecht im internationalen multimodalen Gütertransport (2005) 105, 107; Ramming, Keine Anwendung der CMR auf Teilstrecken einer Multimodal-Beförderung, NJW 2009, 414. Vgl. die Mitteilung der EU-Kommission zu „Güterverkehrslogistik in Europa der Schlüssel zur nachhaltigen Mobilität“, $\operatorname{KOM}(2006) 336$ endg (Nr. 4.2.7.3.: „Zuständigkeiten und Haftung im internationalen Verkehr ergeben sich aus internationalen Übereinkommen. Häufig enthalten diese unterschiedliche Regeln für die einzelnen Verkehrsträger. Die Komplexität und Vielfalt der Regelungen führt zu Reibungsverlusten in multimodalen Verkehrsketten“).

${ }^{20}$ Jesser-Huß, in Handwörterbuch II, 1088; vgl. Laimer, Trasporto multimodale internazionale di merci: novità dalla Corte di Giustizia UE in tema di competenza giurisdizionale, in Pagliarin/Perathoner/Laimer (a cura di), Per un'Europa più unita: Le nuove sfide del diritto del trasporto nazionale ed internazionale (2020) 89 (93).

${ }^{21}$ Übereinkommen der Vereinten Nationen über den internationalen multimodalen Transport von Gütern v. 24.5.1980 (MTC).

${ }^{22}$ Mankowski, in Reithmann/Martiny Rz 6.2092. Die 2009 gezeichneten Rotterdam-Regeln würden zumindest die Beförderung vor und nach der Seestrecke erfassen, sind jedoch ebenfalls mangels ausreichender Ratifikationen bisher nicht in Kraft getreten, Maurer, RabelsZ 81 (2017) 146, 148.

${ }^{23} \mathrm{Zu}$ einem anderen System siehe Weidemann, Emissions- und staufreier alpenquerender Gütertransport: Wachstumspotenziale und -hürden für die Schiene, in diesem Band, Kap. 11.

${ }^{24}$ Siehe etwa Schütz, in Straube/Ratka/Rauter, UGB I ${ }^{4}$ (2009) Art. 2 CMR Rz 1; Bahnsen, in Ebenroth/Boujong/Joost/Strohn, Handelsgesetzbuch ${ }^{3}$ (2015) CMR Art. 2 Rz 8; Jesser-Huß, in Münchener Kommentar zum $\mathrm{HGB}^{3}$ (2014) CMR Art. 2 Rz 4. Jedoch verweist Art. 2 Abs. 1 S. 2 CMR bei Unfällen auf dem Trägerfahrzeug in bestimmten Fällen auf dessen Haftungsrecht, Mankowski, in Reithmann/Martiny Rz 6.2096.
} 
erfasst, jedoch in Abgrenzung zur CMR nur, solange dieser nicht grenzüberschreitend ist. ${ }^{25}$

Um die Auswirkungen fehlenden materiellen Einheitsrechts zu erfassen, muss man sich vergegenwärtigen, dass auch in den nationalen Rechtsordnungen, mit Ausnahme der Niederlande oder etwa Deutschlands, regelmäßig Vorschriften für den multimodalen Transport fehlen, weshalb die jeweilige Rechtsprechung mit den auftretenden praktischen Rechtsfragen umgehen muss. ${ }^{26}$ Dabei können im Wesentlichen zwei große Bewältigungsstrategien unterschieden werden: Entweder gilt ein einheitliches Haftungsregime für den gesamten Transport, unabhängig von einem konkreten Bezug des Streits zu einer spezifischen Teilstrecke (Einheitssystem), ${ }^{27}$ oder es wird das Einheitsrecht herangezogen, das für jene Teilstrecke maßgeblich ist, auf welcher der konkrete Schaden eingetreten ist (Networksystem), ${ }^{28}$ was natürlich dann weitere Schwierigkeiten bereitet, wenn sich - wie in der Praxis häufig beim Containerverkehr - der Schadenseintrittsort nicht aufklären lässt. ${ }^{29}$

\footnotetext{
${ }^{25}$ MüKo-HGB/Freise, CIM Art. $1 \mathrm{Rz} 11 \mathrm{ff}$. Zu anderen Regelungen, die nach einer weiteren Auffassung multimodale Sachverhalte erfassen (z. B. für Art. 18 Abs. 3, Art. 31 WA), Mankowski, in Reithmann/Martiny Rz 6.2105; MüKo-BGB/Martiny, Rom I-VO Art. 5 Rz 147; zu Art. 18 Abs. 3 MÜ siehe etwa Freise, Unimodale transportrechtliche Übereinkommen und multimodale Beförderungen, TranspR 2012, 1.

${ }^{26}$ Siehe auch Perathoner, Il trasporto multimodale nel diritto dell'Unione Europea: un fenomeno trasportistico emergente privo di un'adeguata regolamentazione, in diesem Band, Kap. 3.

${ }^{27}$ Hierzu (auf der Grundlage von Art. 1696 Codice civile i. d. F. Legislativdekret Nr. 286/2005) in Italien Corte di Cassazione v. 6.10.2013 Nr. 18657: ,il trasporto multimodale di cose per via marittima e terrestre, sebbene caratterizzato dall' assoluta prevalenza del tratto marittimo, non rientra nell'ambito della normativa speciale prevista dalla convenzione di Bruxelles del 1924 sulla polizza di carico riguardante il solo contratto che si svolge esclusivamente per via marittima, ma rimane regolato dalla disciplina del codice civile“; anders noch Tribunale Genova v. 12.3.1992 (Absorptionstheorie).
}

${ }^{28}$ Maurer, RabelsZ 81 (2017) 147. Zur österreichischen Rechtsprechung siehe OGH RIS-Justiz RS0062353: „Hat der erteilte Transportauftrag von vornherein die Beförderung mit verschiedenen Beförderungsmitteln zum Gegenstand (Lastkraftwagen, Eisenbahn, Schiff), richtet sich die Ersatzpflicht des mit der Beförderung über die gesamte Strecke beauftragten Frachtführers nach der für das jeweilige Beförderungsmittel geltenden Haftungsordnung (,network-System“)“; OGH $7 \mathrm{Ob}$ 145/10i, SZ 2011/4; 7 Ob 116/17k, wbl 2018, 587: „Das Network-System ist für die Ermittlung der Haftungsordnung bestimmend. Es ist daher bei bekanntem Schadensort auf den zwischen den Parteien des multimodalen Frachtvertrags hypothetisch abgeschlossenen Vertrag über die Beförderung auf derjenigen Teilstrecke abzustellen, auf der der Schaden eingetreten ist. Anstelle des Übernahme- und Auslieferungsorts der multimodalen Beförderung treten der Ort des Beginns und des Endes der betreffenden Teilstrecke“; OGH 7 Ob 2/16v, SZ 2016/131: „Die für die jeweiligen Teilstrecken geltenden Haftungsbestimmungen sind nicht nur dann maßgeblich, wenn von vornherein feststeht, dass der Transport mit verschiedenen Transportmitteln durchgeführt werden soll, sondern auch dann, wenn der Frachtführer in Ausübung seines pflichtgemäßen Ermessens bestimmte Transportarten und -wege gewählt hat“; 7 Ob 45/20y, TranspR 2021, 225.

${ }^{29}$ Mankowski, in Reithmann/Martiny Rz 6.2098. 


\section{Internationale Gerichtszuständigkeit für Klagen aus Verträgen}

Fehlt demnach vorrangiges (Art. 25 Rom I-VO) ${ }^{30}$ international vereinheitlichtes Sachrecht, dann ist das auf den multimodalen Güterbeförderungsvertrag anzuwendende Recht nach dem Kollisionsrecht des jeweils angerufenen Gerichts zu ermitteln. ${ }^{31}$ Die Frage nach dem (den) international zuständigen Gericht(en) ist deshalb freilich vorgelagert, mithin zunächst zu prüfen. ${ }^{32}$ Dafür ist für den Bereich der EU-Mitgliedstaaten nach der Brüssel Ia-Verordnung ${ }^{33}$ vorzugehen. ${ }^{34}$

\subsection{Zuständigkeitsvereinbarung und System objektiver Gerichtsstände im Überblick}

In den praktisch meisten Fällen bestimmen die Parteien des Gütertransportvertrages das für die Streitentscheidung - international oder auch örtlich - zuständige Gericht mittels Vereinbarung (Art. 25 Brüssel Ia-VO), ${ }^{35}$ was auch zu empfehlen ist. Eine solche Gerichtsstandsvereinbarung ist unter Beachtung gewisser Formanforderungen gültig, sofern (1) der für die Anwendung der Verordnung erforderliche Auslandsbezug vorliegt, ${ }^{36}$ (2) die Zuständigkeit eines Gerichts oder der Gerichte eines

\footnotetext{
${ }^{30}$ Musger, in Koziol/Bydlinski/Bollenberger (Hrsg.), Kurzkommentar zum ABGB ${ }^{6}$ (2020) Art. 25 Rom I-VO Rz 2; Simons, in unalex Compendium (2017) Art. 25 Rom I-VO Rz 5. Vgl. in Österreich OGH 7 Ob 5/13f, unalex AT-894 = RdW 2013, 532; in der Schweiz BGE 132 III 626 E. 2.1. ${ }^{31}$ Siehe etwa Verschraegen, Internationales Privatrecht (2012) Rz 1068; Fischer, Ergänzung der CMR durch schweizerisches Recht, TranspR 1995, 424 (431).

${ }^{32}$ Siehe auch Mankowski, Internationale Zuständigkeit und anwendbares Recht - Parallelen und Divergenzen, in Festschrift für Andreas Heldrich (2005) 867 ff. Zum daraus resultierenden Problem des forum shopping siehe allgemein Schwartze, Internationales Forum Shopping mit Blick auf das günstigste Sachrecht, in Festschrift für Bernd von Hoffmann (2011) 415 ff.

${ }^{33}$ Verordnung (EU) 1215/2012 vom 12.12.2012 über die gerichtliche Zuständigkeit und die Anerkennung und Vollstreckung von Entscheidungen in Zivil- und Handelssachen, AB1. 2012 L 351/1. Vgl. dazu etwa Domej, Die Neufassung der EuGVVO. Quantensprünge im europäischen Zivilprozessrecht, RabelsZ 78 (2014) 508.

${ }^{34}$ Siehe im Hinblick auf die Beziehung zwischen Art. 31 CMR und der Brüssel I-Verordnung auf der Grundlage von Art. 71 Brüssel I-VO EuGH 4.9.2014, Rs. C-157, Nickel \& Goeldner Spedition, ECLI:EU:C:2014:2145 = unalex EU-604; 19.12.2013, Rs. C-452/12, Nipponkoa Insurance Co. (Europe), ECLI:EU:C:2013:858 = unalex EU-570; 4.5.2010, Rs. C-533/08, TNT Express Nederland, ECLI:EU:C:2010:243 = unalex EU-222.

${ }^{35}$ Mankowski, Der europäische Erfüllungsortsgerichtsstand des Art. 5 Nr. 1 lit b EuGVVO und Transportverträge, TranspR 2008, 67; Nordmeier, Internationale Gerichtsstandsvereinbarungen nach der EuGVVO n.F., RIW 2016, 331 ff. Siehe jüngst EuGH 7.7.2016, Rs. C-222/15, Höszig, ECLI:EU:C:2016:525.

${ }^{36}$ EuGH 17.11.2011, Rs. C-327/10, Hypoteční banka, ECLI:EU:C:2011:745 (Rz 29 f.); 7.2.2013,
} 
EU-Mitgliedstaats vereinbart ist ${ }^{37}$ und (3) sich die Vereinbarung auf ein bestimmtes Rechtsverhältnis bezieht. ${ }^{38}$

Mangels Gerichtsstandsvereinbarung begründet zunächst Art. 4 Abs. 1 Brüssel Ia-VO die internationale Zuständigkeit der Gerichte im EU-Wohnsitzstaat des Beklagten. ${ }^{39}$ Dabei ist der Wohnsitz natürlicher Personen dem Recht des angerufenen Gerichts zu entnehmen, während der „Wohnsitz“ einer Gesellschaft oder juristischen Person einheitlich europäisch festgelegt wird, und zwar alternativ an dem Ort, an dem sich ihr satzungsmäßiger Sitz, ihre Hauptverwaltung oder ihre Hauptniederlassung befindet (Art. 63 Abs. 1 Brüssel Ia-VO). ${ }^{40}$

Dieser allgemeine Gerichtsstand konkurriert mit den besonderen Zuständigkeiten der Art. 7 bis 9 Brüssel Ia-VO, die Wahlgerichtsstände außerhalb des Wohnsitzstaates der beklagten Partei in einem anderen EU-Mitgliedstaat begründen und auf diese Weise dem Kläger die Wahl einräumen, ob er die Klage beim allgemeinen oder beim besonderen Gerichtsstand einbringen will. ${ }^{41}$ Von den speziellen Zuständigkeitsregelungen für Verbrauchersachen sind Beförderungsverträge ausgenommen (Art. 17 Abs. 3 Brüssel Ia-VO). ${ }^{42}$ Ein nach den europäischen Zuständigkeitsvorschriften unzuständiges Gericht wird grundsätzlich gem. Art. 26 Abs. 1 S. 1 Brüssel Ia-VO zuständig, wenn sich der Beklagte vorbehaltlos auf das Verfahren einlässt. ${ }^{43}$

Rs. C-543/10, Refcomp, ECLI:EU:C:2013:62 (Rz 17); 14.11.2013, Rs. C-478/12, Maletic, ECLI:EU:C:2013:735 (Rz 26).

${ }^{37}$ EuGH 9.11.2000, Rs. C-387/98, Coreck, ECLI:EU:C:2000:606 (Rz 13, 19).

${ }^{38}$ EuGH 7.2.2013, Rs. C-543/10, Refcomp, ECLI:EU:C:2013:62 (Rz 25). Vgl. in Italien z. B. Cass. Civ. sez. un. 7.9.2016 Nr. 17675.

${ }^{39}$ Vgl. EuGH 13.7.2000, Rs. C-412/98, Group Josi, ECLI:EU:C:2000:399.

${ }^{40}$ Siehe Laimer, Internationale gerichtliche Zuständigkeit, in Christandl/Eccher/Gallmetzer/Laimer/Schurr, Handbuch Italienisches Internationales Privatrecht (2019) Rz 2/19. Eine Ausnahme hierzu bildet Art. 24 Nr. 2 S. 2 Brüssel Ia-VO, wonach bei bestimmten Klagen aus dem Gesellschaftsverhältnis für die Bestimmung des Sitzes der Gesellschaft oder juristischen Person das nach den Kollisionsnormen der lex fori berufene Recht heranzuziehen ist.

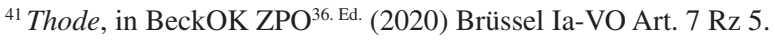

${ }^{42}$ Lurger/Melcher, Handbuch Internationales Privatrecht (2017) Rz 4/92.

${ }^{43}$ Der Einspruch gegen einen Europäischen Zahlungsbefehl stellt in diesem Sinne noch keine Einlassung, mithin keine unmittelbar auf Klageabweisung abzielende Verteidigung dar, EuGH 13.6.2013, Rs. C-144/12, Goldbet Sportwetten, ECLI:EU:C:2013:393 (Rz 43). 


\subsection{Insbesondere: Der Gerichtsstand am Erfüllungsort des Vertrages}

Für vertragliche Ansprüche, also freiwillig eingegangene Verpflichtungen, ${ }^{44}$ ist Art. 7 Nr. 1 lit. a Brüssel Ia-VO von herausragender Bedeutung. ${ }^{45}$ Die Bestimmung eröffnet einen Wahlgerichtsstand, mithin ein alternativ zum allgemeinen Gerichtsstand zur Verfügung stehendes Forum, vor dem Gericht des Ortes, an dem die streitige Verpflichtung erfüllt worden ist oder zu erfüllen wäre. Für die zwei praktisch wichtigsten Vertragstypen, namentlich für den Verkauf beweglicher Sachen ${ }^{46}$ und die Erbringung von Dienstleistungen, sieht Art. 7 Nr. 1 lit. b Brüssel Ia-VO einen unionsrechtlich autonom festgelegten einheitlichen Erfüllungsort dort vor, wo die für den Vertrag charakteristische Leistung, mithin die das gesamte Vertragsverhältnis prägende Verpflichtung, erbracht worden ist oder zu erfüllen wäre, sofern dieser Erfüllungsort in einem EU-Mitgliedstaat liegt. ${ }^{47}$ Diese genannten Merkmale sind in der Folge für die multimodale Güterbeförderung zu bestimmen. ${ }^{48}$

\subsubsection{Die multimodale Güterbeförderung als Dienstleistung}

Der Begriff „Dienstleistung“ gem. Art. 7 Nr. 1 lit. b Brüssel Ia-VO ist unionsrechtlich autonom, also ohne Rückgriff auf mitgliedstaatliche Kriterien, auszulegen. ${ }^{49}$ Nach der Rechtsprechung des EuGH ist eine vertragliche Vereinbarung dann als Dienstleistung i. S. d. Bestimmung einzuordnen, wenn eine Tätigkeit gegen Entgelt geschuldet wird. ${ }^{50}$ Unter den auf diese Weise weit zu verstehenden europäischen Dienstleistungsbegriff, der Verträge mit Tätigkeits- und auch solche mit

\footnotetext{
${ }^{44}$ EuGH 17.06.1992, Rs. C-26/91, Handte, ECLI:EU:C:1992:268 (Rz 15); 05.02.2004, Rs. C-265/02, Frahuil, ECLI:EU:C:2004:77 (Rz 24); 20.01.2005, Rs. C-27/02, Engler, ECLI:EU:C:2005:33 (Rz 51); Gottwald, in MüKo-ZPO5 (2017) Art. 7 Brüssel Ia-VO Rz 3.

${ }^{45}$ Zur Bedeutung des fakultativen Gerichtsstandes für Deliktsklagen am Handlungs- und Erfolgsort nach Art. 7 Nr. 2 Brüssel Ia-VO (dazu etwa Laimer, in Christandl/Eccher/Gallmetzer/Laimer/ Schurr, Handbuch Rz 2/25 ff.) oder etwa des besonderen Gerichtsstandes der Streitgenossenschaft (Art. 8 Nr. 1 Brüssel Ia-VO) für den Bereich des Gütertransports siehe Mankowski, TranspR 2008, 67.

${ }^{46}$ Zur verordnungsautonomen Auslegung des Begriffes EuGH 23.4.2009, Rs. C-533/07, Falco Privatstiftung und Rabitsch, ECLI:EU:C:2009:257 (Rz 19 ff.). Zur Abgrenzung vom Werkvertrag EuGH 25.2.2010, Rs. C-381/08, Car Trim, ECLI:EU:C:2010:90. Siehe Kindler, Der europäische Vertragsgerichtsstand beim Warenkauf im Lichte der Rechtsprechung des Europäischen Gerichtshofes, in Arnold/Lorenz (Hrsg.), Gedächtnisschrift für Hannes Unberath (2015) 255 ff.

${ }^{47}$ Wittwer, in Mayr (Hrsg.), Handbuch des europäischen Zivilverfahrensrechts (2017) Rz 3.273.

${ }^{48}$ Siehe auch Laimer, in Pagliarin/Perathoner/Laimer (Hrsg.), Per un'Europa più unita, 96 ff.

${ }^{49} \mathrm{Vgl}$. Laimer, in Christandl/Eccher/Gallmetzer/Laimer/Schurr, Handbuch Rz 2/22. Grundlegend Kindler, Brussels I and the Principles of Autonomous Interpretation of EU Law, EuLF 2010, $252 \mathrm{ff}$.

${ }^{50}$ Junker, Internationales Zivilprozessrecht ${ }^{3}$ (2016) 96.
} 
Erfolgsverpflichtung umfasst, ${ }^{51}$ sind auch (multimodale) Beförderungsverträge zu subsumieren. ${ }^{52}$

\subsubsection{Erbringungsort der Transportdienstleistung}

Wo liegt nun bei der Gütertransportdienstleistung der Erbringungsort, dem auf diese Weise gerichtsstandsbegründende Kraft zugemessen wird? Bei Dienstleistungsverträgen wird auf den Ort der Dienstleistungen abgestellt, das heißt: Vor Leistungserbringung ist maßgeblich, wo die Dienstleistung nach dem Vertrag hätte erbracht werden müssen; ${ }^{53}$ danach ist der tatsächliche Leistungsort heranzuziehen, sofern er den vertraglichen Bestimmungen entspricht oder die Leistung vom Vertragspartner an diesem Ort vertragsgemäß angenommen worden ist. ${ }^{54}$

Bei Beförderungsverträgen werden nun freilich die wesentlichen Tätigkeiten typischerweise an mehreren Orten erbracht: ${ }^{55}$ Am Ausgangsort muss der Beförderer das Transportmittel bereithalten (zuweilen auch das Gut auf- und einladen), dann folgt der eigentliche Transport, mithin die physische Bewegung der Güter, die am Bestimmungsort der Beförderung abzuliefern sind. ${ }^{56}$

Mit dieser Problematik hatte sich der Europäische Gerichtshof ${ }^{57}$ jüngst in einem Fall auseinanderzusetzen, in dem der multimodale Transport eines RaupenKegelbrechers von Pori (Finnland) nach Sheffield (Vereinigtes Königreich) vereinbart war. Der Brecher wurde zuerst auf der Ladefläche eines LKW von Pori nach Rauma (Finnland) transportiert, wo er abgeladen wurde und mit eigener Maschinenkraft auf ein Schiff fuhr. Nach dem Seetransport zum Hafen Hull (Vereinigtes Königreich) fuhr der Brecher ebenfalls mit eigener Maschinenkraft vom Schiff und wurde dann auf einen zweiten Lastkraftwagen geladen. Schließlich wurde der Brecher von Hull auf dem Landweg abtransportiert, kam aber abhanden, bevor er

\footnotetext{
${ }^{51}$ Mankowski, TranspR 2008, 69.

${ }^{52}$ EuGH 9.7.2009, Rs. C-204/08, Rehder, ECLI:EU:C:2009:439 (Rz 40).

${ }^{53}$ (Auch konkludente) Vereinbarungen über den Erfüllungsort begründen einen Gerichtsstand nach Art. 7 Nr. 1 Brüssel Ia-VO grundsätzlich dann, wenn sie den Erfordernissen des Vertragsstatuts genügen, EuGH 17,1.1980, Rs. C-56/79, Zelger, ECLI:EU:C:1980:15 (Rz 6); 9.6.2011, Rs. C-87/10, Electrosteel Europe, ECLI:EU:C:2011:375 (Rz 22). Jedoch müssen Vereinbarungen, die keinen realen Leistungsort festlegen, sondern lediglich einen Gerichtsstand bestimmen sollen, den von Art. 25 Brüssel Ia-VO für Gerichtsstandsvereinbarungen festgelegten Formvorschriften genügen, EuGH 20.2.1997, Rs. C-106/95, MSG, ECLI:EU:C:1997:70 (Rz 31, 35).

${ }^{54}$ EuGH 11.3.2010, Rs. C-19/09, Wood Floor Solutions Andreas Domberger, ECLI:EU:C:2010:137 (Rz 38, 40).

${ }^{55}$ Vgl. die Schlussanträge des Generalanwalts v. 10.04.2018, Rs. C-88/17, ECLI:EU:C:2018:224 (Rz 28 ff.); Hartenstein, Zur Bestimmung des Gerichtsstands des Erfüllungsorts in der EuGVVO Anmerkung zum Urteil des EuGH vom 11.07.2018 (Rs C-88/17), TranspR 2018, 440.

${ }^{56}$ Mankowski, TranspR 2008, $70 \mathrm{ff}$.

${ }^{57}$ EuGH 11.7.2018, Rs. C-88/17, Zurich Insurance und Metso Minerals, ECLI:EU:C:2018:558. Siehe dazu auch Laimer/Perathoner, Jurisdiction Based on Place of Performance in the Case of a Multimodal Transport. Notes on ECJ 11-07-2018 - C-88/17 - Zurich Insurance plc, Metso Minerals Oy ./. Abnormal Load Services (International) Ltd ( $\rightarrow$ unalex EU-764), EuLF 2 (2019) 39 ff.
} 
seinem Empfänger in Sheffield übergeben werden konnte. Das mit dem Streitfall befasste finnische Gericht hatte dem EuGH im Wesentlichen die Frage zur Vorabentscheidung vorgelegt, wie der Ort bzw. die Orte der Erbringung einer Dienstleistung bestimmt werden, wenn es um einen Vertrag geht, der die Güterbeförderung zwischen Mitgliedstaaten betrifft, und der Transport aus mehreren Teilen besteht, in denen unterschiedliche Transportmittel verwendet werden.

$\mathrm{Zu}$ Verträgen über die Beförderung von Personen im Luftverkehr, und zwar auch bei in Teilstrecken aufgeteilten Flügen ${ }^{58}$ hat der Gerichtshof bereits entschieden, dass die bestimmenden Orte, die eine unmittelbare Verbindung zu den vertragstypischen Dienstleistungen aufweisen, sowohl der Abflugs- als auch der Ankunftsort des Fluges sind, da ,es in solchen Fällen nicht möglich ist, anhand wirtschaftlicher Kriterien einen gesonderten Teil der Leistung auszumachen, der die an einem bestimmten Ort erbrachte Hauptleistung darstellte" ${ }^{59}$ Danach besteht ein Erfüllungsort am Abflug- und am Ankunftsort und der Kläger kann zwischen diesen Orten wählen. ${ }^{60}$

In Weiterführung dieser Vorjudikatur hat der EuGH in Bezug auf die multimodale Güterbeförderung dem vorlegenden finnischen Gericht geantwortet, dass dem Kläger ein Wahlrecht zwischen dem Ausgangsort und dem Bestimmungsort des Transports zukommt. ${ }^{61}$ Beide Orte weisen eine enge Verbindung zum Kern der vertraglichen Leistungspflichten auf. ${ }^{62}$ Zwischenstopps sind dagegen ebenso wenig für den Vertrag prägend wie ein bloßer Umladeort. ${ }^{63}$

Damit ist klargestellt, dass dieses Verständnis des Art. 7 Nr. 1 lit. b Brüssel Ia-VO sowohl für den Personen- als auch für den Gütertransport und dabei für sämtliche Verkehrsträger und eben auch für multimodale Beförderungen gilt. ${ }^{64}$ Die Entscheidung ist im Ergebnis zu begrüßen. ${ }^{65}$ Mit zwei Erfüllungsorten, die dem Kläger zur Wahl stehen, bleibt deren Zahl übersichtlich, sie sind für die Vertragsparteien

\footnotetext{
${ }^{58}$ EUGH 7.3.2018, verb. Rs. C-274/16, C-447/16 und C-448/16, flightright, ECLI:EU:C:2018:160 (Rz 65).

${ }^{59}$ EuGH 9.7.2009, Rs. C-204/08, Rehder, ECLI:EU:C:2009:439 (Rz 40). Siehe i.Ü. jüngst auch EuGH 13.2.2020, Rs. C-606/19, Flightright, ECLI:EU:C:2020:101 (Rz 36): Klagen auf Ausgleichszahlungen wegen Annullierung des letzten Teilflugs können bei Gerichten des Abflugorts des ersten Teilflugs erhoben werden.

${ }^{60}$ Lurger/Melcher, Handbuch Rz 4/92; vgl. EuGH 4.9.2014, Rs. C-157/13, Nickel \& Goeldner Spedition, ECLI:EU:C:2014:2145 (Rz 41).

${ }^{61}$ EuGH 11.7.2018, Rs. C-88/17, Zurich Insurance und Metso Minerals, ECLI:EU:C:2018:558 (Rz 23). Anders noch etwa in Österreich OGH 13.03.1996, 3 Ob 514/94 (,Der Umstand, daß die Enddestination des multimodalen Transportes ein Ort in Österreich war, kann, lagen keine Anknüpfungspunkte vor, für sich allein die inländische Gerichtsbarkeit nicht begründen.").

${ }^{62} \mathrm{Vgl}$. EuGH 11.7.2018, Rs. C-88/17, Zurich Insurance und Metso Minerals, ECLI:EU:C:2018:558 (Rz 20).

${ }^{63}$ Mankowski, TranspR 2018,475; vgl.EuGH 9.7.2009, Rs.C-204/08, Rehder, ECLI:EU:C:2009:439 (Rz 40); a. A. Hartenstein, TranspR 2018, 441.

${ }^{64}$ Vgl. die Schlussanträge des Generalanwalts v. 10.04.2018, Rs. C-88/17, ECLI:EU:C:2018:224 (Rz 48 ff.); siehe Mankowski, TranspR 2018, 474 f.; differenzierend Hartenstein, TranspR 2018, 441.

${ }^{65}$ Siehe auch Laimer, in Pagliarin/Perathoner/Laimer (Hrsg.), Per un'Europa più unita, 98 f.
} 
vorhersehbar und zu beiden Orten hat der Vertrag eine hinreichend enge Verbindung. ${ }^{66}$ Im Übrigen sind auch nach Art. 31 CMR, Art. 46 § 1 CIM und Art. 21 Hamburg Rules sowohl Ausgangs- als auch Bestimmungsort gerichtsstandsbegründend. ${ }^{67}$

\section{Anzuwendendes Recht}

Sodann ist das auf den multimodalen Güterbeförderungsvertrag anzuwendende Recht zu bestimmen und zwar aus der Sicht EU-mitgliedstaatlicher Gerichte nach den Anknüpfungsregeln der Rom I-Verordnung, ${ }^{68}$ die eine Sachnormverweisung anordnet. ${ }^{69}$

\subsection{Rechtswahl}

Nach Art. 3 Rom I-VO sind die Parteien - und davon machen sie in der Praxis auch vielfach Gebrauch - befugt, das auf ihren internationalen Gütertransportvertrag anzuwendende Recht (ausdrücklich oder auch konkludent) ${ }^{70}$ frei zu wählen. ${ }^{71}$ Eine besondere Beschränkung bezüglich der wählbaren Rechte besteht grundsätzlich nicht. ${ }^{72}$ Die gebräuchlichen Verfrachter-AGB wie auch etwa das FIATA Multimodal Transport Bill of Lading erklären das Recht des Staates für maßgeblich, in dem der

\footnotetext{
${ }^{66}$ Schlosser, Internationale Zuständigkeit bei grenzüberschreitenden Transporten mit mehreren Verkehrsmitteln aufgrund eines einzigen Auftrags, IPRax 2019, 23 (24).

${ }^{67}$ Vgl. auch Art. 26(1)(c) MTC; anders hingegen Art. 33(1) MÜ (nur Bestimmungsort), siehe zu alldem Mankowski, TranspR 2008, $72 \mathrm{f}$.

${ }^{68}$ Verordnung (EG) 593/2008 vom 17.6.2008 über das auf vertragliche Schuldverhältnisse anzuwendende Recht, ABl. 2008 L 177/6. Anzuwenden auf ab dem 17.12.2009 geschlossene Verträge, Art. 28 Rom I-VO i. d. F. ABl. 2009 L 309/87; vgl. Leible/Lehmann, Die Verordnung über das auf vertragliche Schuldverhältnisse anzuwendende Recht (,Rom I“), RIW 2008, 528 (531).

${ }^{69} \mathrm{Rühl}$, Rechtswahlfreiheit im europäischen Kollisionsrecht, in Festschrift für Jan Kropholler (2008) 187 (195). Zur grundsätzlich umfassenden Reichweite des Vertragsstatuts siehe etwa Laimer/Schwartze, in Fenyves/Kerschner/Vonkilch, Klang ${ }^{3}$ (2019) § 1045 Rz 24. Unabhängig vom geltenden Vertragsstatut kann das Gericht Eingriffsnormen i. S. d. Art. 9 Rom I-VO beachten; zur Berücksichtigung drittstaatlicher Eingriffsnormen, vgl. Laimer, Rechtsfolgen eines Verstoßes gegen ausländisches Vergaberecht, ZRB 2012, 139; Heindler, Schadenersatz mit Auslandsberührung wegen COVID-19, Zak 2020, 146 (147 ff.). Zum Verstoß gegen den ordre public des Forumstaates (Art. 21 Rom I-VO) Verschraegen, in Rummel (Hrsg.), ABGB-Kommentar ${ }^{3}$ (2004) Art. 16 EVÜ Rz 12

${ }^{70}$ Laimer, in Laimer/Perathoner (Hrsg.), Gesellschaftsrechtliche Nebenvereinbarungen in Europa (2013) 3 (28).

${ }^{71}$ Vgl. auch Laimer, Beschränkung rechtsgeschäftlicher Erfüllungsverpflichtungen (2020) 36 ff. Einschränkungen sind bei Binnen(-markt-)sachverhalten gem. Art. 3 Abs. 3, 4 Rom I-VO zu beachten, vgl. etwa von Hein, in Rauscher, EuZPR/EuIPR III (2016) Art. 3 Rom I-VO Rz 100 ff.

${ }^{72}$ MüKo-BGB/Martiny, Rom I-VO Art. 5 Rz 14.
} 
Verfrachter seinen Sitz hat. ${ }^{73}$ Für bestimmte abspaltbare ${ }^{74}$ Elemente des Vertrages kann gem. Art. 3 Abs. 1 S. 3 Rom I-VO auch eine Teilrechtswahl getroffen werden (, ,épeçage“). ${ }^{75}$

\subsection{Objektive Anknüpfung}

Soweit die Vertragsparteien keine Rechtswahl getroffen haben, ist nach der Sonderanknüpfung in Art. 5 Abs. 1 Rom I-VO das Recht jenes Staates anzuwenden, in dem der Beförderer seinen gewöhnlichen Aufenthalt hat, wenn sich dort auch der Übernahmeort, der Ablieferungsort oder der gewöhnliche Aufenthalt des Absenders befindet ${ }^{76}$ ansonsten das Recht des Staates, in dem der vereinbarte Ablieferungsort liegt. ${ }^{77}$ Ausgangs- und Entladeort sind dabei auf die multimodale Beförderung in ihrer Gesamtheit und nicht etwa auf einzelne Teilstrecken zu beziehen. ${ }^{78}$ Reine Beförderungsverträge sind außerdem gem. Art. 6 Abs. 2 lit. b Rom I-VO vom Anwendungsbereich des Internationalen Verbrauchervertragsrechts ausgenommen, vorbehaltlich einer (hier nicht relevanten) Rückausnahme für Pauschalreisen. ${ }^{79}$

\subsection{Ausweichklausel}

Die objektive Regelanknüpfung des Art. 5 Abs. 1 Rom I-VO steht allerdings unter dem Vorbehalt einer aus der Gesamtheit der Umstände des konkreten Falles herrührenden, ${ }^{80}$ offensichtlich ${ }^{81}$ engeren Verbindung zu einem anderen Staat (Art. 5 Abs. 3 Rom I-VO). ${ }^{82}$ Als denkbares Anwendungsbeispiel wird etwa der Fall genannt, bei

\footnotetext{
${ }^{73}$ Mankowski, in Reithmann/Martiny Rz 6.2100.

${ }^{74}$ Martiny, in Reithmann/Martiny Rz 2.27.

${ }^{75}$ Wendland, in BeckOGK (2020) Rom I-VO Art. 3 Rz 189 ff. Grundlegend Aubart, Die Behandlung der dépeçage im europäischen Internationalen Privatrecht (2013).

${ }^{76}$ Spickhoff, in BeckOK BGB ${ }^{49}$. Ed. (2019) VO (EG) 593/2008 Art. 5 Rn. 7; vgl. etwa BGH RIW 2006, 948; TranspR 2010, 76 = NJW-RR 2010, 247; OLG Düsseldorf VersR 2004, 1479.

${ }^{77}$ Musger, in Koziol/Bydlinski/Bollenberger, Art. 5 Rom I-VO Rz 3; Laimer, Internationaler Gütertransport auf der Strasse zwischen Einheitsprivatrecht und Kollisionsrecht, in Festschrift für Thomas Koller (2018) 485 (497). Zum Vorrang der einheitsrechtlichen Transportrechtskonventionen (Art. 25 Abs. 1 Rom I-VO), soweit das jeweilige Übereinkommen seinerseits überhaupt multimodale Transporte erfasst, siehe bereits oben bei 3. sowie etwa MüKo-BGB/Martiny, Rom I-VO Art. $5 \mathrm{Rz} 147$.

${ }^{78}$ Mankowski, in Reithmann/Martiny Rz 6.2101.

${ }^{79}$ Schwartze, in unalex Compendium (2017) Art. 5 Rom I-VO Rz 5; Schilling, Das Internationale Privatrecht der Transportverträge (2016) $315 \mathrm{ff}$.

${ }^{80}$ Staudinger, in Ferrari/Kieninger/Mankowski u. a., Internationales Vertragsrecht ${ }^{3}$ (2018) VO (EG) 593/2008 Art. 5 Rz 63.

${ }^{81}$ Siehe hierzu Mankowski, in Reithmann/Martiny Rz 6.1896.

${ }^{82}$ MüKo-BGB/Martiny, Rom I-VO Art. 5 Rz 38.
} 
dem die Vertragsparteien ihre Niederlassung in unterschiedlichen Staaten haben und Container sukzessive in verschiedenen Häfen entladen werden sollen, ${ }^{83}$ wo eine Anwendung des Rechts am gewöhnlichen Aufenthalt des Beförderers erwogen wird. ${ }^{84}$

\section{Resümee}

Zusammenfassend kann man sagen, dass die verkehrsmittelorientierte Segmentierung der Regelungen anstelle einer zentralen Rechtsquelle für den multimodalen Transport von großem Nachteil ist. ${ }^{85}$ Die je nach Verkehrsträger unterschiedlichen Sondervorschriften für grenzüberschreitende Beförderungen und die teilweise wiederum verschiedenen nationalen Normen für Binnentransporte bringen insbesondere bei den Haftungsbestimmungen z. T. erhebliche Abweichungen, v. a. unterschiedliche Haftungssummen mit sich. ${ }^{86}$ Eingeschränkte Vorhersehbarkeit des im Streitfall befassten Gerichts und des auf den Beförderungsvertrag anwendbaren Rechts erschwert dem Transportunternehmer naturgemäß die Kalkulation seines Haftungsrisikos und dementsprechend die Versicherbarkeit des Beförderungsgeschäfts. Insgesamt wäre eine einheitsrechtliche Regelung für den multimodalen Gütertransport wünschenswert, m. E. sogar dringend erforderlich. ${ }^{87}$

Dabei hängt die Frage der Anwendbarkeit international vereinheitlichten Sachrechts nicht zuletzt auch von den zur Verfügung stehenden technischen Lösungen ab (Stichwort „Huckepackverkehr“). ${ }^{88} \mathrm{Ob}$ die angestrebte vermehrte Verlagerung des Verkehrs auf emissionsärmere Transportmittel (wie die Schiene) gelingt, hängt aber freilich auch mit der Wirtschaftlichkeit dieser technischen Lösungen zusammen. ${ }^{89}$ Vielleicht können auch in diesem Band vorgestellte Ansätze hierzu einen gewissen Beitrag leisten. ${ }^{90}$

\footnotetext{
${ }^{83}$ Spickhoff, in BeckOK BGB ${ }^{49 . ~ E d . ~(2019) ~ V O ~(E G) ~ 593 / 2008 ~ A r t . ~} 5$ Rn. 19.

${ }^{84}$ Thorn, in Palandt, Bürgerliches Gesetzbuch ${ }^{79}$ (2020) Rz 7; Nielsen, in Ferrari/Leible, Rome I Regulation (2009) 106 f.; vgl. BGH NJW 2009, 3371.

${ }^{85} \mathrm{Vgl}$. auch $\operatorname{KOM}(2006) 336$ endg.

${ }^{86}$ Jesser-Huß, in Handwörterbuch II, 1087 ff.

${ }^{87}$ Zur Rechtsentwicklung auch Mankowski, in Reithmann/Martiny Rz 6.2092.

${ }^{88}$ Siehe oben bei 2 .

${ }^{89}$ Bahnsen, in Ebenroth/Boujong/Joost/Strohn, Handelsgesetzbuch ${ }^{3}$ (2015) CMR Art. 2 Rz 9.

${ }^{90}$ Siehe z. B. Bergmeister, Der Brenner Basistunnel - eine europäische Dimension für Mobilität und Nachhaltigkeit, in diesem Band, Kap. 9; Ehrbar, NEAT in der Schweiz - Versprechungen gehalten? Lehren aus einer über 30-jährigen Geschichte, in diesem Band, Kap. 10; Weidemann, Emissions- und staufreier alpenquerender Gütertransport: Wachstumspotenziale und -hürden für die Schiene, in diesem Band, Kap. 11.
} 
Open Access Dieses Kapitel wird unter der Creative Commons Namensnennung 4.0 International Lizenz (http://creativecommons.org/licenses/by/4.0/deed.de) veröffentlicht, welche die Nutzung, Vervielfältigung, Bearbeitung, Verbreitung und Wiedergabe in jeglichem Medium und Format erlaubt, sofern Sie den/die ursprünglichen Autor(en) und die Quelle ordnungsgemäß nennen, einen Link zur Creative Commons Lizenz beifügen und angeben, ob Änderungen vorgenommen wurden.

Die in diesem Kapitel enthaltenen Bilder und sonstiges Drittmaterial unterliegen ebenfalls der genannten Creative Commons Lizenz, sofern sich aus der Abbildungslegende nichts anderes ergibt. Sofern das betreffende Material nicht unter der genannten Creative Commons Lizenz steht und die betreffende Handlung nicht nach gesetzlichen Vorschriften erlaubt ist, ist für die oben aufgeführten Weiterverwendungen des Materials die Einwilligung des jeweiligen Rechteinhabers einzuholen.

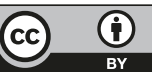

